\title{
Tissue-Specific Transcriptome Analysis in Nodules of Lotus japonicus
}

\author{
Kojiro Takanashi, ${ }^{1}$ Hirokazu Takahashi, ${ }^{2}$ Nozomu Sakurai, ${ }^{3}$ Akifumi Sugiyama, ${ }^{1}$ Hideyuki Suzuki, ${ }^{3}$ \\ Daisuke Shibata, ${ }^{3}$ Mikio Nakazono, ${ }^{2}$ and Kazufumi Yazaki ${ }^{1}$
}

\begin{abstract}
${ }^{1}$ Research Institute for Sustainable Humanosphere, Kyoto University, Gokasho, Uji 611-0011, Japan; ${ }^{2}$ Graduate School of Bioagricultural Sciences, Nagoya University, Nagoya, 464-8601, Japan; ${ }^{3}$ Kazusa DNA Research Institute, Kisarazu, 292-0818, Japan
\end{abstract}

Submitted 20 January 2012. Accepted 9 March 2012.

Legume plants can establish symbiotic nitrogen fixation (SNF) with rhizobia mostly in root nodules, where rhizobia-infected cells are accompanied by uninfected cells in a mosaic pattern. Inside the mature nodules of the legume, carbon and nitrogen nutrients between host plant cells and their resident bacteria are actively exchanged. To elucidate the metabolite dynamics relevant for SNF in nodules, three tissues from a nodule of a model legume, Lotus japonicus, were isolated using laser microdissesction, and transcriptome analysis was done by an oligoarray of 60-mer length representing 21,495 genes. In our tissue-specific profiling, many genes were identified as being expressed in nodules in a spatial-specific manner. Among them, genes coding for metabolic enzymes were classified according to their function, and detailed data analysis showed that a secondary metabolic pathway was highly activated in the nodule cortex. In particular, a number of metabolic genes for a phenylpropanoid pathway were found as highly expressed genes accompanied by those encoding putative transporters of secondary metabolites. These data suggest the involvement of a novel physiological function of phenylpropanoids in SNF. Moreover, five representative genes were selected, and detailed tissue-specific expression was characterized by promoter- $\beta$-glucuronidase experiments. Our results provide a new data source for investigation of both nodule differentiation and tissue-specific physiological functions in nodules.

Nitrogen, an essential element for a living organism, circulates biologically and chemically on the earth. Biological nitrogen fixation represents enzymatic conversion of atmospheric nitrogen to ammonium, which is an important process to ensure nitrogen availability for organisms. This process is mostly managed by symbiotic nitrogen fixation (SNF) established by rhizobia in specialized legume organs called nodules, which develop from root cortical cells after infection of rhizobia in a species-specific manner.

Leguminosae is one of the most successful families of land plants that acquire nitrogen nutrition because SNF enables legumes to utilize atmospheric nitrogen even in nitrogen-poor soils. Due to the function of SNF, legumes are a rich source of proteins and amino acids and, thus, many legume species have

Corresponding author: K. Yazaki; Telephone: +81-774-38-3617; Fax: +81-774-38-3623; E-mail: yazaki@rish.kyoto-u.ac.jp

* The $e$-Xtra logo stands for "electronic extra" and that four supplementary tables and one supplementary figure are published online. been widely cultivated in agriculture as crops and in green fertilizers as well.

Establishment of SNF is initiated from the release of signal compounds (e.g., flavonoids) from legume roots, followed by Nod factor excretion from rhizobia (Lerouge et al. 1990; Peters et al. 1986; Redmond et al. 1986). After perception of signal compounds, rhizobia penetrate into cortical cells through an infection thread, which successively causes the cortical cell division. During nodule developments, both plant cells and rhizobia differentiate concertedly, resulting in the formation of both infected and uninfected plant cells in a nodule with a pavement pattern (Brewin 1991). When the nodule matured and SNF occurred, fixed nitrogen was supplied to the plant from the rhizobia and, in turn, the plant provided reduced carbon and all other nutrients necessary for symbiotic bacteria (White et al. 2007).

A number of transcriptome analyses (e.g., time-course analysis after inoculation of rhizobia and microarray analysis combined with metabolic profiling) were performed to understand global changes in gene expression in both plants and rhizobia. These studies provided comprehensive profiles of gene expression during SNF (Colebatch et al. 2004; Kouchi et al. 2004; Küster et al. 2004). In brief, these reports demonstrated that the gene expression pattern in the early steps of rhizobium-legume interactions is considerably different from that in subsequent nodule development stages. These reports also revealed that the nodule had a distinct metabolic phenotype that reflected changes in gene expression for related metabolic enzymes. However, little is still known about how these gene expressions as well as the dynamic changes of metabolites are regulated in a tissuespecific manner in nodules. Infected cells and uninfected cells are arranged in a mosaic in the infection zone of nodules and, thus, allotments in cellular functions regulated by different genes in the nodules should exist. To understand tissue-specific gene expressions and to predict metabolite dynamics in nodules, in this study, we have conducted laser microdissection (LM) combined with a microarray analysis, and characterized the compartmentalization and regulation of the metabolic pathway in nodule cells in a model legume, Lotus japonicus. We have isolated three tissues from the nodule: the nodule infection zone (IFZ), the nodule parenchyma (NP), and the nodule cortex, including the nodule vascular bundle (NC). These three tissues were then subjected to microarray analysis.

\section{RESULTS AND DISCUSSION}

Microarray analysis and its validation.

To perform the LM microarray of three different tissues in the nodules of L. japonicus, we prepared cross sections of the 
nodules at mature stages (14 to 21 days postinoculation). The three dissections mentioned above (IFZ, NP, and NC) were isolated from the nodule sections using the Veritas Laser Microdissection System (Fig. 1A through E). Because differentiated infected cells exist with uninfected cells in a mosaic pattern and, thus, precise separation of these cell types is technically difficult, we excised IFZ as a mixture of both infected and uninfected cells (Fig. 1C). For NP, we cut off the cell layers adjacent to the infection zone (Fig. 1D). The nodule inner cortex and the nodule vascular bundle were collected as NC (Fig. 1E). The total RNA extracted from the LM-isolated cells was then labeled with Cy3, and subjected to the 44,000 L. japonicus oligoarrays with 21,495 features of 60 -mer oligonucleotides.
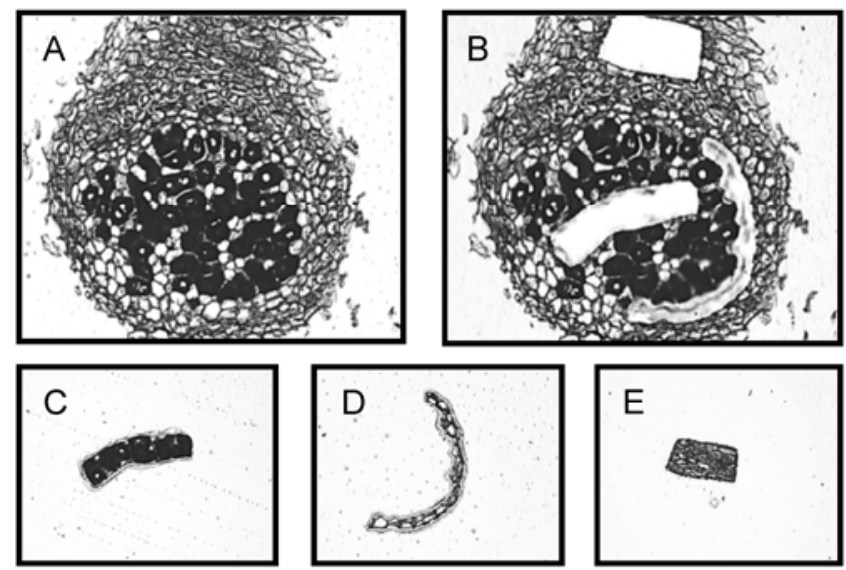

$\mathrm{F}$

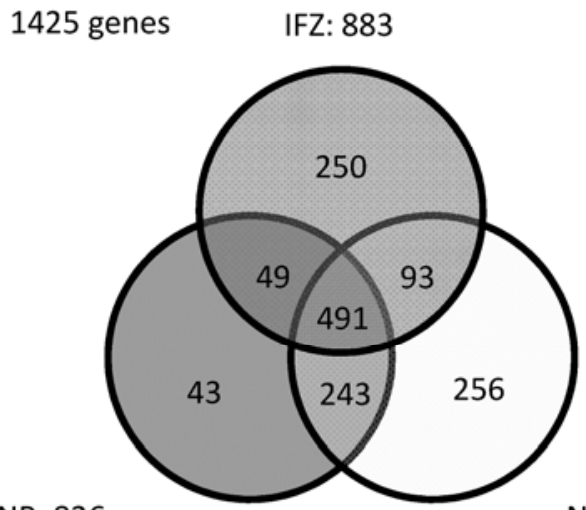

NP: 826

NC: 1083

Fig. 1. Isolation of three tissues from the cross-sections of Lotus japonicus nodules using laser microdissection (LM). Cross-section of a nodule $\mathbf{A}$, before and $\mathbf{B}$, after LM. C, Infection zone (IFZ); D, nodule parenchyma (NP); and E, nodule inner cortex (NC). F, Number of genes for which expression intensities were higher than 2.0, for each tissue.
The microarray experiments were repeated at least three times in each tissue using independently isolated RNA samples.

To identify the genes highly expressed in each tissue, we applied a selection cutoff (expression intensity $\geq 2.0$ ) to obtain 1,425 transcripts as the expressed genes in the LM-isolated cells. Of these, 250 genes were identified as IFZ-specific genes, whereas 43 and 256 genes were NP and NC specific, respectively (Fig. 1F). To evaluate the inter-tissue variation, a correlated analysis with all pairwise samples was performed. The inter-replicate correlation coefficients of IFZ were 0.7 to 0.84, while the inter-tissue coefficients between IFZ and the other two tissues were between -0.18 and 0.56 , indicating that the gene expression profile in IFZ was distinct from the other two tissues. The inter-tissue correlation coefficients between $\mathrm{NP}$ and NC (0.51 to 0.84$)$ were similar to the inter-replicate correlation coefficients ( 0.71 to 0.82 and 0.79 to 0.93 , respectively). These results suggested that NP and NC have similar physiological functions. We then performed principal components analysis (PCA) to visualize the expression variations of three tissues (Supplementary Fig. S1). IFZ are located in a distinct area from other tissues as suggested by the correlation analysis. Although NP and NC were plotted in a similar position, they were separately clustered on the first component, which contains $70 \%$ of the total variance.

To identify differentially regulated genes among these tissues, we performed a one-way analysis of variance (ANOVA) with a post hoc Tukey's range test. Among 1,425 genes, 644 genes were confirmed by the ANOVA procedure with a significance level of 0.05 . Next, we employed a post-ANOVA procedure (Tukey's range test; $P \leq 0.01$ ) to identify 424 genes as being differentially regulated.

To confirm this expression profile biochemically, putative promoter regions for five representative genes, including three metabolic enzymes and two transporters, were isolated and promoter-reporter analyses were performed (Table 1; Fig. 2). Expression of a 2-hydroxyisoflavanone synthase (GA05118), which is expected to be an NC-specific gene from our microarray analysis, was detected only in the nodule vascular bundle, as expected (Fig. 2A to D). Such tissue-specific expression analysis by promoter- $\beta$-glucuronidase (GUS) experiments was also done with other genes (i.e., GA05422, GA01242, and so on) to confirm the validation of tissue specificity in the LM transcriptome (Fig. 2E to L).

\section{Functional classification of metabolic genes expressed in nodules.}

Among 1,425 transcripts, 263 (18.5\%) genes were coded for metabolic enzymes and categorized according to their biochemical function (Table 2; Supplementary Table S2). In a survey of those genes for a variety of metabolic pathways, the number of genes involved in primary metabolism was almost the same among all three tissues; however, genes related to secondary metabolisms (e.g., phenylpropanoid and terpenoid

Table 1. List of genes analyzed by promoter:: $\beta$-glucuronidase experiments

\begin{tabular}{|c|c|c|c|c|c|c|}
\hline \multirow[b]{2}{*}{ Probe ID $^{b}$} & \multirow[b]{2}{*}{ Gene ID $^{c}$} & \multicolumn{3}{|c|}{ Expression level $^{\mathrm{a}}$} & \multirow[b]{2}{*}{$P$ value $^{\mathrm{d}}$} & \multirow[b]{2}{*}{ Annotation } \\
\hline & & IFZ & NP & NC & & \\
\hline GA05118 & chr4.CM0432.2900.r2.m & -3.03 & -0.86 & 2.35 & 4.7E-02 & 2-Hydroxyisoflavanone synthase \\
\hline GA05422 & chr6.CM0367.720.r2.d & -2.78 & 3.43 & 4.08 & $6.3 \mathrm{E}-03$ & 1-Aminocyclopropane-1-carboxylate oxidase \\
\hline GB06367 & chr2.LjB15M17.30.r2.m & 1.25 & 2.04 & 2.47 & $2.2 \mathrm{E}-02$ & Alanine glyoxylate aminotransferase \\
\hline GA01242 & chr6.CM0245.360.r2.m & 2.93 & -0.51 & -3.76 & $1.1 \mathrm{E}-02$ & Purine permease \\
\hline GD09405 & chr3.CM0208.260.r2.m & 1.69 & 2.81 & 2.77 & 4.4E-02 & Metal ion transmembrane transporter \\
\hline
\end{tabular}

${ }^{a}$ IFZ = infection zone, $\mathrm{NP}=$ nodule parenchyma, and $\mathrm{NC}=$ nodule cortex.

${ }^{\mathrm{b}}$ Lotus japonicus microarray probe data.

c L. japonicus genome assembly build 2.5 .

${ }^{\mathrm{d}} P$ values were obtained from analysis of variance. 
biosynthesis) were found in higher frequency in NP and NC compared with IFZ (Table 2). We then analyzed those genes in more detail according to the category of metabolism.

\section{Sugar metabolism.}

Genes mediating sugar breakdown were highly expressed in nodules. These included most metabolic genes responsible for the enzymatic reaction steps from sucrose to phosphoenolpyruvate (PEP), and the expression levels of most genes were not significantly different among the three tissues. Two enzymes directly related to PEP metabolism-that is, pyruvate kinase (PK, EC 2.7.1.40) that produces pyruvate and PEP carboxylase (PEPC, EC 4.1.1.31) that produces oxaloacetate from PEPwere also found in our analysis. The expression of PK was found only in NP and NC, whereas PEPC was detected in all three tissues. In a similar pattern to PK, two pyruvate decarboxylases (EC 4.1.1.1) and one of three pyruvate dehydrogenases (EC 1.2.4.1), which convert pyruvate to acetaldehyde and acetyl-CoA, respectively, were also strongly expressed in NP and $\mathrm{NC}$, suggesting that the pyruvate metabolism in the nodule is mainly conducted in NP and NC. Because pyruvate plays a pivotal role as a metabolic precursor for many biosynthetic pathways, such as those for fatty acids and terpenoids, high gene expression for the pyruvate metabolism in NP and NC suggests the activation of these metabolic pathways in these tissues. Indeed, many genes in fatty acid metabolism, such as acetyl-CoA carboxylase (EC21238), were highly expressed in NP and NC.

\section{Malate dehydrogenase.}

Malate is used as the primary carbon source for bacteroid metabolism and SNF (Day and Copeland 1991), and transporters that deliver dicarboxylates to the bacteroids have been biochemically characterized using isolated soybean symbiosome (Udvardi and Day 1997; Udvardi et al. 1988). Two malate dehydrogenases (MDH, EC 1.1.1.37) were detected in the nodule from our microarray analysis. One belongs to cytosolic $\mathrm{MDH}$, which is involved in the malate-aspartate shuttle, and the other is a nodule-enhanced MDH (neMDH), which shares high amino acid similarity with alfalfa and pea neMDH (87 and 85\%, respectively) (Fedorova et al. 1999; Miller et al. 1998). The pea neMDH expressed in the $\mathrm{N}_{2}$-fixing zone in an indeterminate nodule, and alfalfa neMDH are known to convert oxaloacetate into malate rather than its reverse reaction. The Lotus neMDH expressed also in an $\mathrm{N}_{2}$-fixing zone in the determinate nodule, indicating that the Lotus neMDH may also be involved in producing malate from oxaloacetate to supply to bacteroids. Because the subcellular localizations of neMDH were unknown, clarification of their subcellular localizations will provide us with a new metabolic flow in IFZ.

\section{Nitrogen metabolism.}

Genes involved in nitrogen metabolism such as ammonium assimilation and asparagine synthesis were found in the transcriptome. Asparagine is the major nitrogen compound translocated from nodules to the Lotus plant (Vance et al. 1987). Thus, it is in conformity with the high expression of aspartate aminotransferases (AAT, EC 2.6.1.1) and asparagine synthases (AS, EC 6.3.5.4) in IFZ because they facilitate rapid synthesis of this amino acid, although glutamine synthetase (GS, EC 6.3.1.2) expressed in IFZ was not found in the present experiment. It is to be noted that an AS (LjAS2) was highly expressed in three tissues, while two AAT were strongly induced in IFZ, and an NC-specific GS was also detected.

As genes involved in amino acids metabolism, we found more than 30 genes that were expressed in nodules. Alanine is synthesized by alanine aminotransferase (AlaAT, EC 2.6.1.2) from pyruvate and aspartate, while alanine-glyoxylate amino- transferase (AlaGAT, EC 2.6.1.44) mediates the reverse reaction. Alanine accumulation in nodules was previously reported (Colebatch 2004) and our data indicate that both AlaAT and AlaGAT were expressed in nodules in an NP- and NC-specific fashion. This may be due to the restricted pyruvate metabolism in NP and NC, as mentioned above. In addition, three enzymes which mediate the last step of amino acid biosynthesis pathways - that is, cysteine synthase (EC 2.5.1.47), methionine synthase (EC; 1.16.1.8), and threonine synthase (EC 4.2.3.1) were highly expressed in IFZ.

\section{Secondary metabolism.}

Phenylpropanoids and flavonoids are biosynthesized from phenylalanine/tyrosine through several enzymatic steps. The biosynthesis route from phenylalanine/tyrosine to p-coumaroyl$\mathrm{CoA}$ is common for phenylpropanoids and flavonoids, and two out of three genes involved in this pathway - that is, phenylalanine ammonia-lyases (EC 4.3.1.24) and cinnamate 4-hydroxylase (EC 1.14.13.11) - were upregulated in NP and NC. $p$-Coumaroyl-CoA is then converted to $p$-coumaroyl shikimate by shikimate $O$-hydroxycinnamoyltransferase (EC 2.3.1.133) for phenylpropanoid synthesis. Almost all genes involved in phenylpropanoid synthesis were highly expressed in NP and NC. Phenylpropanoids provide the main building blocks of both suberin and lignin, which function as a physical barrier or mechanical support at the cell wall. Because nodule vascular bundles are developed through the nodule's inner cortex, where lignin accumulation is necessary, expression of genes in the phenylpropanoid pathway at NP will be responsible for the synthesis of the nodule vascular bundle. Hartmann and associates (2002) reported that the nodule infection zone of the broad bean (Vicia faba), which forms indeterminate nodules, was surrounded by an apoplastic suberin barrier that functions as a gas diffusion barrier. Suberin is composed of phenolic components (phenylpropanoid) and aliphatic components (Bernards 2002), and genes necessary for the biosynthesis of aliphatic precursors such as fatty acids were also found in NP in this experiment, suggesting that the suberin barrier also plays an important role in the determinate nodule around the infection zone, which keeps the oxygen level low in IFZ.

It is noteworthy that a set of genes for the flavonoid biosynthesis pathway was highly expressed in NP. In legume plants, flavonoids are used as signal compounds in the initial step for the establishment of symbiosis with rhizobia, and they also serve phytoalexin molecules as a defense response against phytopathogenic organisms. In addition to direct interaction with microbes, flavonoids are necessary for forming the nodule by

Table 2. Number of metabolic enzyme genes classified by the functional category ${ }^{\mathrm{a}}$

\begin{tabular}{lcrr}
\hline Category & IFZ & NP & NC \\
\hline Starch and sucrose metabolism & 7 & 5 & 6 \\
Fructose and mannose metabolism & 3 & 3 & 3 \\
Pentose phosphate pathway & 4 & 4 & 5 \\
Glycolysis/gluconeogenesis & 7 & 9 & 10 \\
Citrate cycle/glyoxylate and & 6 & 6 & 7 \\
$\quad$ dicarboxylate metabolism & 21 & 21 & 26 \\
Amino acids metabolism & 3 & 5 & 5 \\
Purine/pyrimidine metabolism & 19 & 21 & 26 \\
Lipid metabolism & 3 & 2 & 1 \\
Porphyrin metabolism & 4 & 11 & 28 \\
Phenylpropanoid biosynthesis & 0 & 3 & 4 \\
Terpenoid biosynthesis & 2 & 5 & 6 \\
Glutathione metabolism & 2 & 5 & 6 \\
Cell wall modification & 49 & 54 & 63 \\
Others metabolism & 130 & 154 & 196 \\
Total & & \\
\hline
\end{tabular}

${ }^{a} \mathrm{IFZ}=$ infection zone, $\mathrm{NP}=$ nodule parenchyma, and $\mathrm{NC}=$ nodule cortex. 
inhibition of polar auxin transport at the site of the rhizobia infection, especially in indeterminate nodules such as Medicago truncatula and white clover (Wasson et al. 2006; Zhang et al. 2009). In the nodulation process of the determinate legume, the flavonoid pathway seemed to be activated in at least four different stages: first, in a nitrogen nutrient deficiency condition to release flavonoids as signal compounds (Sugiyama et al. 2007); second, shortly after Nod factor perception as defense-related genes (Nakagawa et al. 2011); third, during nodule primordia development (Deguchi et al. 2007; Kouchi et al. 2004); and, fourth, in mature nodules without known physiological functions (Colebatch et al. 2004; Kouchi et al. 2004; Shimada et al. 2007). Some of those flavonoid-related genes were also identified as glutathione responsive genes, and then seemed to mediate a part of vestitol biosynthesis, an isoflavan phytoalexin of Lotus japonicus and other legume plants (Shimada et al. 2007), suggesting a function of flavonoid derivatives as a chemical barrier against other microbes in the nodules. In addition, our promoter::GUS analysis of the 2-hydroxyisoflavanone synthase (EC 1.14.13.86), which mediates a key step of isoflavonoid synthesis (Shimada et al. 2000), showed that the synthase was expressed at the nodule cortex in a young nodule, at the rootnodule junction, and also at nodule vascular bundles in a matured nodule (Fig. 2A and B). This expression pattern was similar to that of the soybean GH3 promoter in Lotus nodules (Takanashi et al. 2011), indicating that flavonoids function as a regulator of auxin flow also in determinate nodules.

\section{Transporter genes expressed in the nodule.}

Several transporters relevant for SNF were identified using a genetic approach and "omics" studies (Colebatch et al. 2004;
Kouchi et al. 2004; Krusell et al. 2005; Pelissier et al. 2004; Wienkoop and Saalbach 2003). However, an overall understanding of membrane transport systems functioning in nodules is still very limited. In our microarray analysis, 67 genes encoding transporters have been found. As carbohydrate transporters, two sugar transporters and three polyol transporters were detected, which may function in sugar distribution in SNF. A dicarboxylate carrier was also found in IFZ. In the literature, only one transporter, LjSUT4, was characterized as a sugar transporter in the Lotus nodule (Flemetakis et al. 2003; Reinders et al. 2008), and no malate transporter that should have been localized at the symbiosome has been identified yet. It will be interesting to reveal the carbon flux in the nodule by characterizing transport functions and subcellular localization of these transporters identified in Supplementary Table S3. They are classified into different transporter families, as described below.

\section{ABC proteins.}

ATP-binding cassette $(\mathrm{ABC})$ proteins constitute one of the largest transporter families in plants, with approximately 120 members each in Arabidopsis, rice, and L. japonicus (Jasinski et al. 2003; Sanchez-Fernandez et al. 2001; Sugiyama et al. 2006). Because ABC proteins are involved in a wide range of biological processes and function as transporters, channel regulators, and molecular switches, characterization of their physiological roles will point to new aspects in the regulatory mechanisms of SNF. Nine ABC proteins were found in our experiment, which included an auxin-transport $\mathrm{ABC}$ protein, LjABCB1 (Takanashi et al. 2012). Three half-size A-type ABC proteins $(\mathrm{ABCA})$ were expressed in the nodule; in particular,
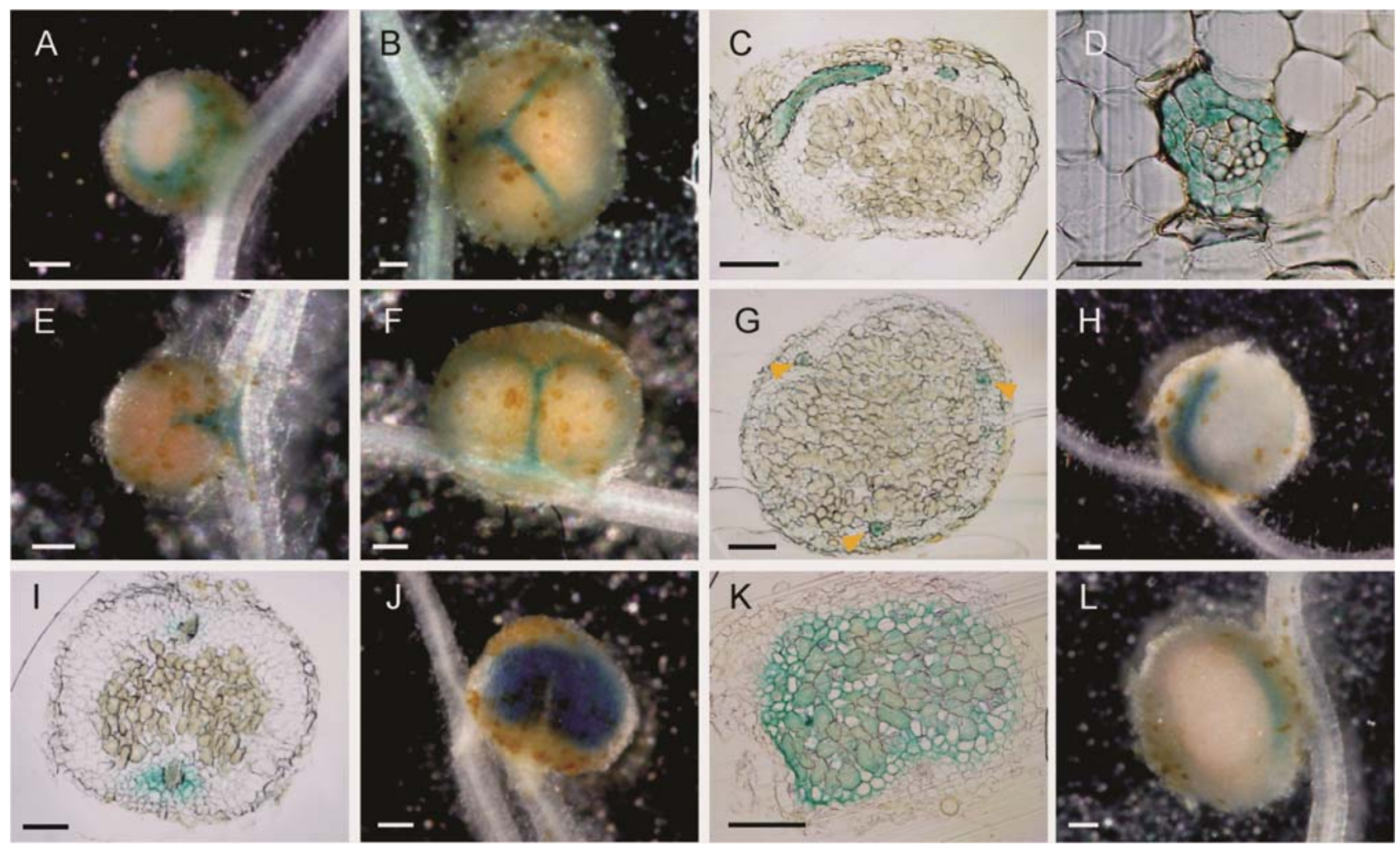

Fig. 2. $\beta$-Glucuronidase (GUS) staining of promoter::GUS transformants. A and $\mathbf{E}$, Young and $\mathbf{B}, \mathbf{F}, \mathbf{H}, \mathbf{J}$, and $\mathbf{L}$, mature nodules; $\mathbf{C}, \mathbf{D}, \mathbf{G}, \mathbf{I}$, and $\mathbf{K}$, crosssections of mature nodules. A through D, 2-Hydroxyisoflavanone synthase; strong expression was observed in NP and NC in the young nodule (A), whereas only the nodule vascular bundle was stained in the mature nodule (B and C). E through $\mathbf{G}$, Expression of 1-aminocyclopropane-1-carboxylate oxidase; GUS expression was detected only in the nodule vascular bundle through nodulation. H and I, Alanine glyoxylate aminotransferase; GUS expression was observed around nodule vascular bundles. $\mathbf{J}$ and $\mathbf{K}$, Purine permease; GUS staining showed infection zone-specific expression during nodulation. L, Metal ion transmembrane transporter; GUS staining was detected at root-nodule junction. Bars $=200 \mu \mathrm{m}$ (A through C and E through L) and $10 \mu \mathrm{m}$ (D). 
in IFZ. Recently, we have found that RNA interference lines of an Arabidopsis ABCA member showed altered lipid composition (K. Yazaki and C. Forestier unpublished data), suggesting a lipid transport by these nodule-expressed ABCA proteins in IFZ. Three G-type ABC proteins (ABCG) were also expressed in the L. japonicus nodule. Of these, an ABCG member (GA01187) was previously identified as being one of the legume-specific $\mathrm{ABC}$ proteins because of its high redundancy in the L. japonicus genome (Sugiyama et al. 2006) and the induced expression in the underground part of L. japonicus after inoculation with M. loti. (Kouchi et al. 2004). Its Nicotiana plumbaginifolia homolog, $\mathrm{NpABC} 1$, showed efflux activity for an endogenous anti-fungal diterpene, sclareol (Jasinski et al. 2001; Stukkens et al. 2005), and an Arabidopsis homolog, AtABCG40, has been recently reported to transport a sesquiterpene plant hormone, abscissic acid (Kang et al. 2010), suggesting that this Lotus ABC protein also transports terpenoid in the nodule.

\section{Multidrug and toxic compound extrusion proteins.}

Multidrug and toxic compound extrusion (MATE) proteins provide another large transporter family in the plant genome (e.g., 56 MATE orthologs were found in the Arabidopsis genome) (Li et al. 2002). Reported plant MATE proteins are clustered into three groups according to their amino acid sequence similarity, which seemed to reflect their preference for transport substrates (e.g., flavonoids, alkaloids, and citrate). In our microarray experiment, two MATE proteins were identified and one (GA04042) belonged to the cluster of flavonoid transporters. This MATE protein was mainly ex- pressed in NP, where the phenylpropanoid pathway was actively expressed (discussed above), suggesting that this MATE protein is involved in the accumulation of flavonoids in NP. In recent years, two MATE proteins belonging to the flavonoid transporter clade were reported from another legume plant, $M$. truncatula, and, as expected, they showed a transport function for a flavonoid substrate (Zhao and Dixon 2009; Zhao et al. 2011). However, both proteins function in aerial parts and no flavonoid transporter in the nodule was reported. This Lotus MATE found in our study is the first identification of a putative flavonoid transporter in the nodule, and the biochemical and physiological characterization of this MATE will provide detailed functions of flavonoids in nodules.

Another MATE protein (GA00902) found in this experiment was a member of the clade for citrate transporters, which was highly expressed in IFZ. In fact, a dicarboxilic acid, malate, is an important organic acid for SNF as a carbon source of bacteroid, whereas the involvement of the citrate in SNF is unknown. This MATE subfamily is reported to be involved in $\mathrm{Al}$ detoxification and $\mathrm{Fe}$ translocation by effluxing citrate at the root epidermis and in the root vascular bundle, respectively, in Arabidopsis, rice, and sorghum (Durrett et al. 2007; Furukawa et al. 2007; Magalhaes et al. 2007; Yokosho et al. 2009). Fe is an essential metal for SNF because leghemoglobin, the most abundant protein in a nodule, requires Fe for heme biosynthesis (Appleby 1984). As an important Fe donor protein for leghemoglobin, ferritin is localized in infected cells at the beginning of SNF but the localization shifts into uninfected cells with time (Lucas et al. 1998). The Fe transport mechanism be-

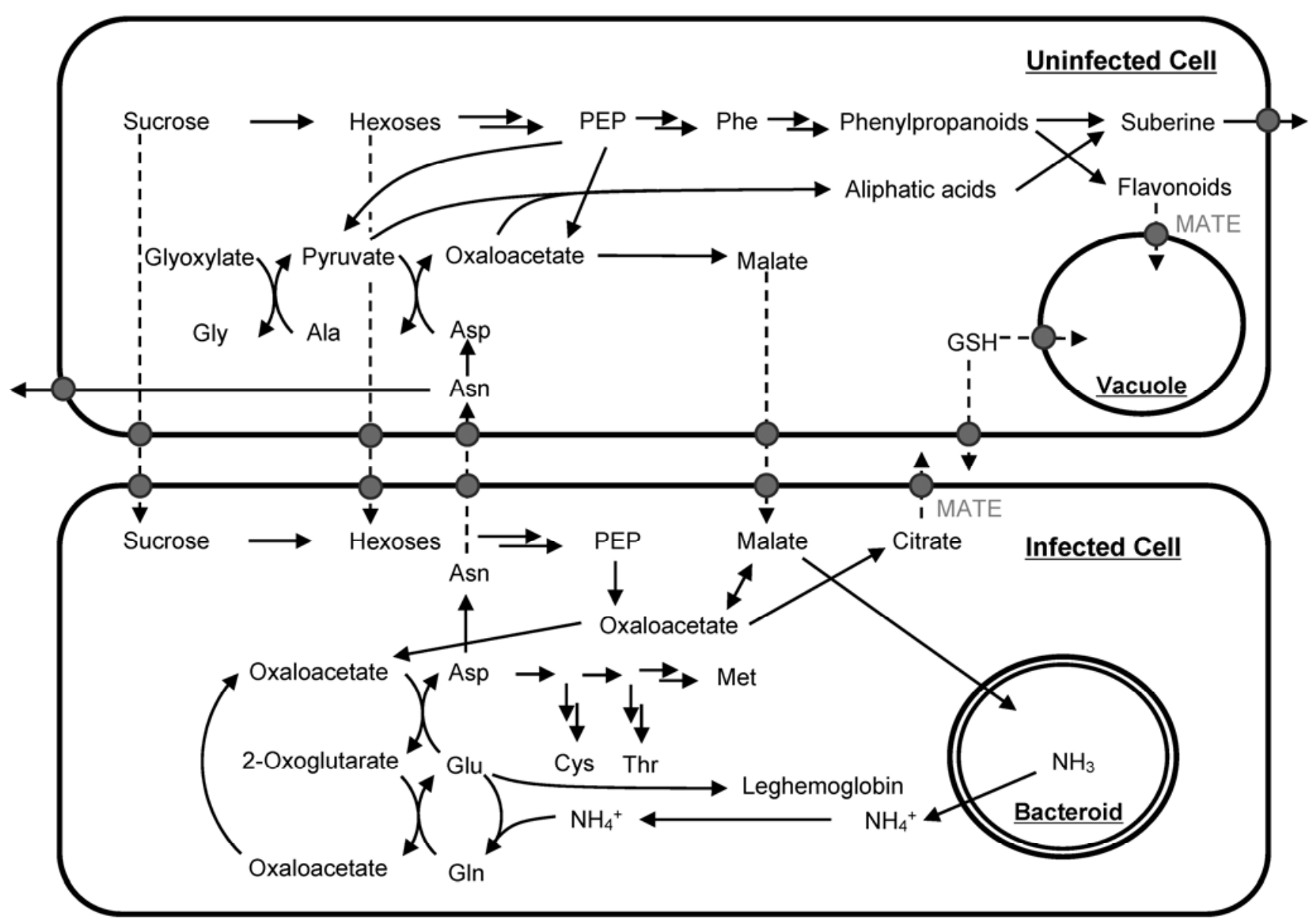

Fig. 3. Proposed model of metabolite dynamics in Lotus nodule. Subcellular localizations were not considered. Putative metabolite flows are shown by the dashed lines.

Vol. 25, No. 7, 2012 / 873 
tween infected and uninfected cells (i.e., the mechanism of $\mathrm{Fe}$ supply from ferritin to leghemoglobin) still remains to be clarified. Whether this MATE protein is, by transporting citrate, involved in Fe translocation that supports SNF in L. japonicus is expected to be proved.

\section{Concluding remarks.}

In this study, we have performed a tissue-specific transcriptome analysis using LM in L. japonicus nodules. We have provided a comprehensive data source of a gene expression profile with detailed spatial information in the nodules (Fig. 3). LM is highly useful for characterization of the transcriptome in specialized tissues, which are difficult to separate by usual biochemical treatments; that is, the separation of stelar cells and cortical cells in maize root (Rajhi et al. 2011) and precise clipping of the infection site of powdery mildew at the Arabidopsis leaf (Chandran et al. 2010). Through this method, we excised three different tissues from L. japonicus nodules, and analyzed the gene expression with a microarray to find that at least 1,425 genes were expressed in Lotus nodules in a tissue-specific manner. An interesting finding in our transcriptome analysis in Lotus nodules is the high expression of genes relevant for the flavonoid biosynthesis and pyruvate metabolism in NP and NC (Fig. 3). We also found a putative flavonoid transporting-MATE member, which was expressed at the same cells where flavonoid biosynthetic genes were highly expressed, suggesting that biosynthesis and accumulation of flavonoids in the nodule is mediated in the same cells (Fig. 3). Many regulation mechanisms for biological events during SNF (e.g., differentiation of infected and uninfected cells and the senescence process) are still largely unknown. Our gene list provides good candidate genes to characterize their functions further in depth, and their subcellular locations are also of high interest. The tissue-specific transcriptome data presented in this study will widely help the relevant research community to elucidate the molecular mechanism of SNF processes.

\section{MATERIALS AND METHODS}

\section{Plant materials and growth conditions.}

L. japonicus MG-20 Miyakojima was used in this work. Plants were grown in a cultivation chamber under a day-night cycle of 16 and $8 \mathrm{~h}$, respectively, at temperatures of $23^{\circ} \mathrm{C}$. Seed were surface sterilized with a $1 \%$ sodium hypochloride solution for $10 \mathrm{~min}$, rinsed five times with sterile distilled water, then germinated on a water agar plate $(0.8 \%)$. Five-dayold seedlings were transferred to a sterile vermiculite with liquid 1/2 B\&D medium (Broughton and Dilworth 1971) in a plant box. After 2 days, plants were inoculated with $M$. loti MAFF303099, which had been cultured overnight in a TY medium at $28^{\circ} \mathrm{C}$.

\section{Isolation of three tissues.}

Nodules at 14 to 21 days postinoculation were fixed in Farmer's fixative (ethanol/acetate, $3: 1$ ) overnight at $4^{\circ} \mathrm{C}$. Dehydration and paraffin embedding were performed as described by using a microwave processor (Inada and Wildermuth 2005; Takahashi et al. 2010). Paraffin-embedded sections were prepared at a thickness of $16 \mu \mathrm{m}$ and mounted on PEN membrane glass slides (Molecular Devices, Menlo Park, CA, U.S.A.) for LM, as described by Takahashi and associate (2010). To remove the paraffin, slides were immersed in Histoclear II (National Diagnostics, Atlanta, GA, U.S.A.) for 5 min twice, and then, were air dried completely at room temperature. LM was performed using the Veritas laser microdissection system LCC1704 (Molecular Devices).

\section{Microarray experiment.}

Total RNAs were extracted from LM cells with a PicoPure RNA isolation kit (Molecular Devices) according to the instruction manual. The extracted total RNA was quantified with a Quant-iT RiboGreen RNA reagent and kit (Invitrogen, Carlsbad, CA, U.S.A.) according to the manufacturer's instructions. The quality of extracted RNA was assessed using an RNA 6000 Pico kit on an Agilent 2100 Bioanalyzer (Agilent Technologies, Santa Clara, CA, U.S.A.) as described previously (Takahashi et al. 2010). A L. japonicus 44,000 oligo microarray (Agilent Technologies), which contains 21,495 oligonucleotides, was synthesized based on nucleotide sequence and expressed sequence tag data. Fluorescent probe labeling, using the oligo-dT-T7 strand-specific amplification method, and hybridization were performed using a Quick Amp labeling kit according to the manufacturer's instructions (Agilent Technologies). The analyses were performed in three biological replicates. The hybridized slides were scanned using a DNA microarray scanner G2505C (Agilent Technologies) and signal intensities were extracted by Feature Extraction software (version 10.5.1.1; Agilent Technologies). A complete set of microarray data was deposited to the Gene Expression Omnibus repository under accession number GSE34753.

\section{Microarray data analysis.}

All analyses were performed using R software. Raw expression data were transformed binary log values, and applied to quantile normalization across all microarrays to make uniform the signal intensities for each array. The cutoff level $\geq 2.0$ was employed for the genes expressed in nodules according to previous reports (Flemetakis et al. 2004; Kouri et al. 2009). PCA and correlation analysis were used to evaluate inter-replicate variance.

Gene IDs were identified from the L. japonicus genome assembly build 2.5 by BLASTN similarity searches using the probe sequences, which were downloaded from the Kazusa $L$. japonicus custom oligo DNA microarray as queries. The crosshybridized probe sequences were removed from the list of Supplementary Table 1 . The putative genes coding for metabolic enzymes were categorized in reference to a KEGG database. To obtain $P$ values, one-way ANOVA was performed, and wherever group differences were confirmed by the ANOVA with a significance level of 0.05 , Tukey's range test $(P \leq 0.01)$ was employed to detect differentially regulated genes.

\section{Histochemical analysis \\ of promoter-GUS transformant of $L$. japonicus.}

Fragments of approximately $2.0 \mathrm{~kb}$ upstream of the start codon of genes were amplified from genome DNA of MG-20 using Phusion High-Fidelity DNA Polymerase (Finnzymes) with the sets of gene-specific primers (Supplementary Table 4). The polymerase chain reaction products were cloned into pBI101 (Clontech), then introduced into L. japonicus using hairy-root transformation mediated by Agrobacterium rhizogenes LBA1334, as previously reported (Kumagai and Kouchi 2003). The transformed plants were inoculated with $M$. loti and grown in a cultivation chamber under the condition mentioned above. GUS staining of the nodule was performed as described previously (Takanashi et al. 2011).

\section{ACKNOWLEDGMENTS}

This work was supported, in part, by a Grant-in-Aid for Scientific Research (number 21027022 to K. Yazaki), Research Fellowships for Young Scientists from the Japan Society for the Promotion of Science (number 09J00170 to K. Takanashi), and the New Energy and Industrial Technology Development Organization, Japan (number P02001 to N. Sakurai, H. 
Suzuki, and D. Shibata). We thank the National Bioresource Project (L. japonicus, Glycine max) for providing seed of $L$. japonicus, H. Kamakura (The University of Tokyo, Japan) for her technical assistance in preparing LM samples, Y. Nagamura, and R. Motoyama (National Institute of Agrobiological Sciences, Japan) for the microarray experiment, M. den DulkRas (Leiden University, Netherlands) for A. rhizogenes LBA1334, and T. Nakagawa (Meiji University, Japan) for his useful advice.

\section{LITERATURE CITED}

Appleby, C. A. 1984. Leghemoglobin and rhizobium respiration. Annu. Rev. Plant Physiol. 35:443-478.

Bernards, M. A. 2002. Demystifying suberin. Can. J. Bot. 80:227-240.

Brewin, N. J. 1991. Development of the legume root nodule. Annu. Rev. Cell Biol. 7:191-226.

Broughton, W. J., and Dilworth, M. J. 1971. Control of leghaemoglobin synthesis in snake beans. Biochem. J. 125:1075-1080.

Chandran, D., Inada, N., Hather, G., Kleindt, C. K., and Wildermuth, M. C. 2010. Laser microdissection of Arabidopsis cells at the powdery mildew infection site reveals site-specific processes and regulators. Proc. Natl. Acad. Sci. U.S.A. 107:460-465.

Colebatch, G., Desbrosses, G., Ott, T., Krusell, L., Montanari, O., Kloska, S., Kopka, J., and Udvardi, M. K. 2004. Global changes in transcription orchestrate metabolic differentiation during symbiotic nitrogen fixation in Lotus japonicus. Plant J. 39:487-512.

Day, D. A., and Copeland, L. 1991. Carbon metabolism and compartmentation in nitrogen-fixing legume nodules. Plant Physiol. Biochem. 29:185-201.

Deguchi, Y., Banba, M., Shimoda, Y., Chechetka, S. A., Suzuri, R., Okusako, Y., Ooki, Y., Toyokura, K., Suzuki, A., Uchiumi, T., Higashi, S., Abe, M., Kouchi, H., Izui, K., and Hata, S. 2007. Transcriptome profiling of Lotus japonicus roots during arbuscular mycorrhiza development and comparison with that of nodulation. DNA Res. 14:117-133.

Durrett, T. P., Gassmann, W., and Rogers, E. E. 2007. The FRD3-mediated efflux of citrate into the root vasculature is necessary for efficient iron translocation. Plant Physiol. 144:197-205.

Fedorova, M., Tikhonovich, I. A., and Vance, C. P. 1999. Expression of Cassimilating enzymes in pea (Pisum sativum L.) root nodules. In situ localization in effective nodules. Plant Cell Environ. 22:1249-1262.

Flemetakis, E., Dimou, M., Cotzur, D., Efrose, R. C., Aivalakis, G., Colebatch, G., Udvardi, M., and Katinakis, P. 2003. A sucrose transporter, LjSUT4, is up-regulated during Lotus japonicus nodule development. J. Exp. Bot. 54:1789-1791.

Flemetakis, E., Efrose, R. C., Desbrosses, G., Dimou, M., Delis, C., Aivalakis, G., Udvardi, M. K., and Katinakis, P. 2004. Induction and spatial organization of polyamine biosynthesis during nodule development in Lotus japonicus. Mol. Plant-Microbe Interact. 17:1283-1293.

Furukawa, J., Yamaji, N., Wang, H., Mitani, N., Murata, Y., Sato, K., Katsuhara, M., Takeda, K., and Ma, J. F. 2007. An aluminum-activated citrate transporter in barley. Plant Cell Physiol. 48:1081-1091.

Hartmann, K., Peiter, E., Koch, K., Schubert, S., and Schreiber, L. 2002. Chemical composition and ultrastructure of broad bean (Vicia faba L.) nodule endodermis in comparison to the root endodermis. Planta 215:14-25.

Inada, N., and Wildermuth, M. C. 2005. Novel tissue preparation method and cell-specific marker for laser microdissection of Arabidopsis mature leaf. Planta 221:9-16.

Jasinski, M., Stukkens, Y., Degand, H., Purnelle, B., Marchand-Brynaert, J., and Boutry, M. 2001. A plant plasma membrane ATP binding cassette-type transporter is involved in antifungal terpenoid secretion. Plant Cell 13:1095-1107.

Jasinski, M., Ducos, E., Martinoia, E., and Boutry, M. 2003. The ATPbinding cassette transporters: structure, function, and gene family comparison between rice and Arabidopsis. Plant Physiol. 131:1169-1177.

Kang, J., Hwang, J. U., Lee, M., Kim, Y. Y., Assmann, S. M., Martinoia, E., and Lee, Y. 2010. PDR-type ABC transporter mediates cellular uptake of the phytohormone abscisic acid. Proc. Natl. Acad. Sci. U.S.A. 107:2355-2360.

Kouchi, H., Shimomura, K., Hata, S., Hirota, A., Wu, G.J., Kumagai, H., Tajima, S., Suganuma, N., Suzuki, A., Aoki, T., Hayashi, M., Yokoyama, T., Ohyama, T., Asamizu, E., Kuwata, C., Shibata, D., and Tabata, S. 2004. Large-scale analysis of gene expression profiles during early stages of root nodule formation in a model legume, Lotus japonicus. DNA Res. 11:263-274.

Kouri, E. D., Labrou, N. E., Garbis, S. D., Kalliampakou, K. I., Stedel, C., Dimou, M., Udvardi, M. K., Katinakis, P., and Flemetakis, E. 2009. Molecular and biochemical characterization of the parvulin-type PPIases in Lotus japonicus. Plant Physiol. 150:1160-1173.

Krusell, L., Krause, K., Ott, T., Desbrosses, G., Kramer, U., Sato, S.,
Nakamura, Y., Tabata, S., James, E. K., Sandal, N., Stougaard, J., Kawaguchi, M., Miyamoto, A., Suganuma, N., and Udvardi, M. K. 2005. The sulfate transporter SST1 is crucial for symbiotic nitrogen fixation in Lotus japonicus root nodules. Plant Cell 17:1625-1636.

Kumagai, H., and Kouchi, H. 2003. Gene silencing by expression of hairpin RNA in Lotus japonicus roots and root nodules. Mol. Plant-Microbe Interact. 16:663-668.

Küster, H., Hohnjec, N., Krajinski, F., El Yahyaoui, F., Manthey, K. Gouzy, J., Dondrup, M., Meyer, F., Kalinowski, J., Brechenmacher, L., van Tuinen, D., Gianinazzi-Pearson, V., Puhler, A., Gamas, P., and Becker, A. 2004. Construction and validation of cDNA-based Mt6kRIT macro- and microarrays to explore root endosymbioses in the model legume Medicago truncatula. J. Biotechnol. 108:95-113.

Lerouge, P., Roche, P., Faucher, C., Maillet, F., Truchet, G., Prome, J. C., and Denarie, J. 1990. Symbiotic host-specificity of Rhizobium meliloti is determined by a sulfated and acylated glucosamine oligosaccharide signal. Nature 344:781-784.

Li, L. G., He, Z. Y., Pandey, G. K., Tsuchiya, T., and Luan, S. 2002. Functional cloning and characterization of a plant efflux carrier for multidrug and heavy metal detoxification. J. Biol. Chem. 277:5360-5368.

Lucas, M. M., Van de Sype, G., Herouart, D., Hernandez, M. J., Puppo, A., and de Felipe, M. R. 1998. Immunolocalization of ferritin in determinate and indeterminate legume root nodules. Protoplasma 204:61-70.

Magalhaes, J. V., Liu, J., Guimaraes, C. T., Lana, U. G. P., Alves, V. M. C., Wang, Y. H., Schaffert, R. E., Hoekenga, O. A., Pineros, M. A., Shaff, J. E., Klein, P. E., Carneiro, N. P., Coelho, C. M., Trick, H. N., and Kochian, L. V. 2007. A gene in the multidrug and toxic compound extrusion (MATE) family confers aluminum tolerance in sorghum. Nat. Genet. 39:1156-1161.

Miller, S. S., Driscoll, B. T., Gregerson, R. G., Gantt, J. S., and Vance, C. P. 1998. Alfalfa malate dehydrogenase (MDH): molecular cloning and characterization of five different forms reveals a unique noduleenhanced MDH. Plant J. 15:173-184.

Nakagawa, T., Kaku, H., Shimoda, Y., Sugiyama, A., Shimamura, M., Takanashi, K., Yazaki, K., Aoki, T., Shibuya, N., and Kouchi, H. 2011. From defense to symbiosis: limited alterations in the kinase domain of LysM receptor-like kinases are crucial for evolution of legume-Rhizobium symbiosis. Plant J. 65:169-180.

Pelissier, H. C., Frerich, A., Desimone, M., Schumacher, K., and Tegeder, M. 2004. PvUPS1, an allantoin transporter in nodulated roots of French bean. Plant Physiol. 134:664-675.

Peters, N. K., Frost, J. W., and Long, S. R. 1986. A plant flavone, luteolin, induces expression of Rhizobium meliloti nodulation genes. Science 233:977-980.

Rajhi, I., Yamauchi, T., Takahashi, H., Nishiuchi, S., Shiono, K. Watanabe, R., Mliki, A., Nagamura, Y., Tsutsumi, N., Nishizawa, N. K., and Nakazono, M. 2011. Identification of genes expressed in maize root cortical cells during lysigenous aerenchyma formation using laser microdissection and microarray analyses. New Phytol. 190:351-368.

Redmond, J. W., Batley, M., Djordjevic, M. A., Innes, R. W., Kuempel, P. L., and Rolfe, B. G. 1986. Flavones induce expression of nodulation genes in rhizobium. Nature 323:632-635.

Reinders, A., Sivitz, A. B., Starker, C. G., Gantt, J. S., and Ward, J. M. 2008. Functional analysis of LjSUT4, a vacuolar sucrose transporter from Lotus japonicus. Plant Mol. Biol. 68:289-299.

Sanchez-Fernandez, R., Davies, T. G. E., Coleman, J. O. D., and Rea, P. A. 2001. The Arabidopsis thaliana ABC protein superfamily, a complete inventory. J. Biol. Chem. 276:30231-30244.

Shimada, N., Akashi, T., Aoki, T., and Ayabe, S. 2000. Induction of isoflavonoid pathway in the model legume Lotus japonicus: molecular characterization of enzymes involved in phytoalexin biosynthesis. Plant Sci. 160:37-47.

Shimada, N., Sato, S., Akashi, T., Nakamura, Y., Tabata, S., Ayabe, S. I., and Aoki, T. 2007. Genome-wide analyses of the structural gene families involved in the legume-specific 5-deoxyisoflavonoid biosynthesis of Lotus japonicus. DNA Res. 14:25-36.

Stukkens, Y., Bultreys, A., Grec, S., Trombik, T., Vanham, D., and Boutry, M. 2005. NpPDR1, a pleiotropic drug resistance-type ATP-binding cassette transporter from Nicotiana plumbaginifolia, plays a major role in plant pathogen defense. Plant Physiol. 139:341-352.

Sugiyama, A., Shitan, N., Sato, S., Nakamura, Y., Tabata, S., and Yazaki, K. 2006. Genome-wide analysis of ATP-binding cassette (ABC) proteins in a model legume plant, Lotus japonicus: comparison with Arabidopsis ABC protein family. DNA Res. 13:205-228.

Sugiyama, A., Shitan, N., and Yazaki, K. 2007. Involvement of a soybean ATP-binding cassette-type transporter in the secretion of genistein, a signal flavonoid in legume-Rhizobium symbiosis. Plant Physiol. 144:2000-2008.

Takahashi, H., Kamakura, H., Sato, Y., Shiono, K., Abiko, T., Tsutsumi, N., Nagamura, Y., Nishizawa, N. K., and Nakazono, M. 2010. A method 
for obtaining high quality RNA from paraffin sections of plant tissues by laser microdissection. J. Plant. Res. 123:807-813.

Takanashi, K., Sugiyama, A., and Yazaki, K. 2011. Involvement of auxin distribution in root nodule development of Lotus japonicus. Planta 234:73-81.

Takanashi, K., Sugiyama, A., Sato, S., Tabata, S., and Yazaki, K. 2012. LjABCB1, an ATP-binding cassette protein specifically induced in uninfected cells of Lotus japonicus nodules. J. Plant Physiol. 169:322-326.

Udvardi, M. K., and Day, D. A. 1997. Metabolite transport across symbiotic membranes of legume nodules. Annu. Rev. Plant Physiol. 48:493523

Udvardi, M. K., Price, G. D., Gresshoff, P. M., and Day, D. A. 1988. A dicarboxylate transporter on the peribacteroid membrane of soybean nodules. FEBS (Fed. Eur. Biochem. Soc.) Lett. 231:36-40.

Vance, C. P., Reibach, P. H., and Pankhurst, C. E. 1987. Symbiotic properties of Lotus pedunculatus root nodules induced by Rhizobium loti and Bradyrhizobium sp. (Lotus). Physiol. Plant. 69:435-442.

Wasson, A. P., Pellerone, F. I., and Mathesius, U. 2006. Silencing the flavonoid pathway in Medicago truncatula inhibits root nodule formation and prevents auxin transport regulation by rhizobia. Plant Cell 18:16171629.

White, J., Prell, J., James, E. K., and Poole, P. 2007. Nutrient sharing between symbionts. Plant Physiol. 144:604-614.

Wienkoop, S., and Saalbach, G. 2003. Proteome analysis. Novel proteins identified at the peribacteroid membrane from Lotus japonicus root nodules. Plant Physiol. 131:1080-1090.

Yokosho, K., Yamaji, N., Ueno, D., Mitani, N., and Ma, J. F. 2009. OsFRDL1 is a citrate transporter required for efficient translocation of iron in rice. Plant Physiol. 149:297-305.

Zhang, J., Subramanian, S., Stacey, G., and Yu, O. 2009. Flavones and flavonols play distinct critical roles during nodulation of Medicago truncatula by Sinorhizobium meliloti. Plant J. 57:171-183.

Zhao, J., and Dixon, R. A. 2009. MATE transporters facilitate vacuolar uptake of epicatechin 3'-O-glucoside for proanthocyanidin biosynthesis in Medicago truncatula and Arabidopsis. Plant Cell 21:2323-2340.

Zhao, J., Huhman, D., Shadle, G., He, X. Z., Sumner, L. W., Tang, Y., and Dixon, R. A. 2011. MATE2 mediates vacuolar sequestration of flavonoid glycosides and glycoside malonates in Medicago truncatula. Plant Cell 23:1536-1555.

\section{AUTHOR-RECOMMENDED INTERNET RESOURCES}

Kazusa DNA Research Institute Lotus japonicus genome assembly build 2.5: www.kazusa.or.jp/lotus/index.html

Kazusa Lotus japonicus custom oligo DNA microarray: www.kazusa.or.jp/pgtech/LjArray

Kyoto Encyclopedia of Genes and Genomes (KEGG) database: www.genome.jp/kegg

R Project for Statistical Computing website: www.r-project.org 